Pacific Journal of Mathematics

ON A PROBLEM OF J. F. RITA 


\section{ON A PROBLEM OF J. F. RITT}

\section{Kathleen B. O'KeEFE}

In the Ritt algebra $R\{u, v\}=R\left(u_{0}, v_{0}, u_{1}, v_{1}, u_{2}, v_{2}, \cdots\right)$ where the derivation is such that $y_{i}^{\prime}=y_{i+1}$ for $y=u$ or $v$, consider the differential ideal $\Omega=[u v]=\left((u v),(u v)_{1},(u v)_{2}, \cdots\right)$. Let $P=u_{i_{1}} \cdots u_{i_{m}} v_{j_{1}} \cdots v_{j_{n}}$ be a power product in $u, v$ and their derivatives. For sufficiently large $q$, it is known that $P^{q} \equiv 0[u v]$. Power products of the form $u_{i} v_{j}$ are of particular interest; one of J. F. Ritt's unsolved problems is to find the smallest $q$ such that $\left(u_{i} v_{j}\right)^{q} \equiv 0[u v]$. The purpose of this paper is to solve this problem in the special case $i=1$. The main theorem is: The smallest $q$ such that $\left(u_{1} v_{j}\right)^{q} \equiv 0[u v]$ is $2+j$. Part of the solution involves generalizing some results of D. G. Mead and part is an application of the well-known reduction process of $H$. Levi.

Basic Notions, H. Levi's reduction process [1] starts with replacing a factor $u_{a} v_{b}$ of $P=u_{i_{1}} \cdots u_{i_{m}} v_{j_{1}} \cdots v_{j_{n}}=u_{a} v_{b} Q$ by the other terms in $(u v)_{a+b}$. Thus we obtain the congruence

$$
P \equiv-\sum_{\substack{i=0 \\
i \neq a}}^{a+b} \frac{\left(\begin{array}{c}
a+b \\
i
\end{array}\right)}{\left(\begin{array}{c}
a+b \\
a
\end{array}\right)} u_{i} v_{a+b-i} Q[u v] .
$$

Repeated substitutions of this kind eventually permit $P$ to be congruent to a linear combination of terms which are not in [uv], unless all of the coefficients are zero. The unwieldy coefficients involved in this reduction process are simplified by $M$-congruences which may be introduced in the following way: Let $S$ be the set

$$
\left(a_{0}, a_{1}, a_{2}, \cdots ; b_{0}, b_{1}, b_{2}, \cdots ; c_{0}, c_{1}, c_{2}, \cdots\right)
$$

and $A$ the free algebra on $S$ over $R$. Define an (algebra) homomorphism $h: A \rightarrow R\{u, v\}$ by $h(r)=r$ if $r \in R, h\left(a_{i}\right)=u_{i} / i !, h\left(b_{i}\right)=v_{i} / i !$, and $h\left(c_{i}\right)=(u v)_{i} / i !$. There exists an isomorphism of $A / K$ into $R\{u, v\}$, where $K$ is the kernel of $h$ and this isomorphism becomes a differential isomorphism by defining $\left(y_{i}\right)_{1}=(i+1) y_{i+1}$ for $y_{i}=a_{i}, b_{i}$ or $c_{i}$ and leaving the derivative in $R$ unchanged. Now consider the algebra obtained by replacing $a_{i}, b_{i}$, and $c_{i}$ by $u_{i}, v_{i}$ and $(u v)_{i}$ respectively. The relation

Received June 19, 1964 and in revised form December 2, 1964. This paper was written while the author held a fellowship from the American Association of University Women. 


$$
(u v)_{n}=\sum_{i=0}^{n}\left(\begin{array}{c}
n \\
i
\end{array}\right) u_{i} v_{n-i}
$$

has become

$$
(u v)_{n}=\sum_{i=0}^{n} u_{i} v_{n-i} .
$$

An $M$-congruence in the original algebra $R\{u, v\}$ is an ordinary congruence in this isomorphic image of $R\{u, v\}$.

Throughout this paper, the notation and terminology of [1] and [2] are used. All congruences are $M$-congruences and are taken modulo $[u v]$.

2. Minimum weight sequences. Let $R\{u, v\}$ be a $R i t t$ algebra in the indeterminates $u$ and $v, Q=[u v]$ the differential ideal generated by the form $X=u v$, and $P=u_{i_{1}} \cdots u_{i_{m}} v_{j_{1}} \cdots v_{j_{n}}$ a power product of weight $w=\sum_{1}^{m} i_{p}+\sum_{1}^{n} j_{q}$, and signature $(m, n)$. For each $\left(m_{i}, n_{i}\right)$, with $1 \leqq m_{i} \leqq m$ and $1 \leqq n_{i} \leqq n$, take the minimum weight of all factors of $P$ of signature $\left(m_{i}, n_{i}\right)$. Subtract $m_{i} n_{i}$ from that minimum. The result is one number for each pair $\left(m_{i}, n_{i}\right)$. The set of these excess weights is called the weight matrix of $P$. A theorem of $H$. Levi [1] says that if there is a negative entry in the weight matrix of $P$, then $P$ is in $[u v]$. To reduce the number of excess weights that need to be computed, the idea of an ordered power product and the concept of a minimum weight sequence are introduced.

An ordered power product $Q$ is of the form: $Q=f_{1} f_{2} \cdots f_{n}$, where for each $i, i=1,2, \cdots, n, f_{i}=u_{d(i)}$ or $f_{i}=v_{d(i)}$. For each $m \leqq n, Q_{m}=f_{1} \cdots f_{m}$ is an initial factor of $Q$, of, say, signature $(k, l)$ and weight $w$. The excess weight of $Q_{m}$ is $e_{m}=w-k l$. Thus $Q$ has a sequence of excess weights of its initial factors, $\left\{e_{1}, \cdots, e_{n}\right\}$. The sequence $\left\{e_{1}, \cdots, e_{n}\right\}$ is called the initial factor weight sequence of $Q$.

Let $P$ be any power product of signature $(m, n)$. Let $Q_{d}$ be any factor of $P$ of signature $(k, l), k+l=d$ and smallest excess weight, $w_{d}$.

Definition 2.1. The sequence $\left\{w_{1}, \cdots, w_{m+n}\right\}$ is the minimum weight sequence of $P$.

A simple method of constructing $\left\{w_{1}, \cdots, w_{m+n}\right\}$ is shown next.

Lemma 2.2. $Q_{d} \mid Q_{d+1}$. 
Proof. The $u$ (and $v$ ) subscripts of $Q_{t}, t=1, \cdots, m+n$, form a nondecreasing sequence of natural numbers; call them $r(i)$ and $s(i)$ respectively. Among all pairs $(x, y), x+y=d$, by hypothesis,

$$
\sum_{1}^{x} r(i)+\sum_{1}^{y} s(i)-x y
$$

is a minimum for $x=k$ and $y=l$. We claim that the corresponding minimum for $d+1$ is attained by either $x=k+1, y=l$ or $x=k$, $y=l+1$. Consider the two expressions:

$$
\begin{aligned}
& \sum_{1}^{k+j+1} r(i)+\sum_{1}^{l-j} s(i)-(k+j+1)(l-j) \\
& \sum_{1}^{k+j} r(i)+\sum_{1}^{l-j+1} s(i)-(k+j)(l-j+1) .
\end{aligned}
$$

Subtract (2) from (1). The difference

$$
\Delta=r(k+j+1)+k+2 j-s(l-j+1)-l
$$

should be positive for (1) to be larger for $j>0$ than for $j=0$.

Since $w_{d}$ is a minimum,

$$
r(k+1)+(k+1) \geqq s(l)+l .
$$

Therefore, if $j>0,2 j>1, r(k+j+1) \geqq r(k+1)$ and $s(l-j) \leqq s(l)$. Thus $\Delta>0$ and $Q_{d+1}$ must be $Q_{d} u_{r}$ or $Q_{d} v_{s}$.

LEMMA 2.3. $Q_{d+1}$ is $Q_{d} u_{r}$ or $Q_{d} v_{s}$ according as

$$
s-r+l-k>0
$$

or

$$
s-r+l-k<0 .
$$

(For $s-r+l-k=0$, either $u_{r}$ or $v_{s}$ yields a minimum.)

Proof. Let $P=Q_{d} u_{r}$ and the excess weight of $P$,

$$
e_{d+1}=w_{d}+r-(k+1) l .
$$

Replace $u_{r}$ by $v_{s}$ and call the new excess weight $e_{d+1}^{*}$,

$$
e_{d+1}^{*}=v_{d}+s-(k)(l+1) .
$$

The change gives a smaller excess weight if and only if

$$
e_{d \div 1}^{*}-e_{d \div 1}<0,
$$

or

$$
s-r+l-k<0 .
$$


The following theorems are simple consequences of Lemmas 2.2 and 2.3, and Levi's theorem.

Theorem 2.4. For any power product $P$, there is an ordered power product $P^{*}$ whose initial factor weight sequence is the minimum weight sequence of $P$.

THEOREM 2.5. If an entry in the minimum weight sequence of $P$ is negative, then $P \equiv 0[u v]$.

3. Generalized $\alpha$-terms. If $P=u_{i_{1}} \cdots u_{i_{m}} v_{j_{1}} \cdots v_{j_{n}}$ and $j_{q} \geqq m$ for $q=1,2, \cdots, n$, then $P$ is called an $\alpha$-term. For example, there is exactly one $\alpha$-term of signature $(m, n)$ and weight $m n$, namely $u_{0}^{m} v_{m}^{n}$. The initial factor weight sequence of $u_{0}^{m} v_{m}^{n}$ is its minimum weight sequence and consists entirely of zeros. In this section, a study is made of all power products with minimum weight sequences consisting entirely of zeros.

Definition 3.1. A minimum weight sequence consisting entirely of zeros is called a zero-sequence.

DEFINITION 3.2. A power product whose minimum weight sequence is a zero-sequence is called a generalized $\alpha$-term.

The power product $P=u_{i_{1}} \cdots u_{i_{m}} v_{j_{1}} \cdots v_{j_{n}}$ may be symbolically written $P=U V$. The degree of $P, d(P)$, is the sum of the degrees of $U$ and $V, d(P)=m+n$. The weight of $P, w(P)$, is the sum of the weights of $U$ and $V, w(P)=w(U)+u(V) . \quad w(U)$ is called the $u$-weight of $P$.

Lemma 3.3. Let $P=U V$ be a generalized $\alpha$-term of degree $d=m+n$. Then $P$ has either $u_{n}$ or $v_{m}$ as a factor, but not both $u_{n}$ and $v_{m}$ as factors.

Proof. By Theorem 2.4, let $Q$ be an initial factor of $P$ such that $d(Q)=d(P)-1$ and the excess weight of $Q$ is zero. By Lemma 2.3, either

$$
Q u_{r}=P
$$

or

$$
Q v_{s}=P \text {. }
$$

If (1) holds, then $w(Q)=m n-r$. Also, $w(Q)=(m-1) n$, hence 
$r=n . \quad$ If (2) holds, by similar reasoning, $m=s$.

Suppose that $u_{n}$ and $v_{m}$ are both factors of $P$. Then let $T=P / u_{n} v_{m}$. The excess weight of $T$ is -1 and $P$ can not be a generalized $\alpha$-term.

THEOREM 3.4. If $P$ and $P^{*}$ are generalized $\alpha$-terms of degree $d$ such that $P=U V$ and $P^{*}=U V^{*}$, then $V=V^{*}$.

Proof. The proof is by induction on $d$. For $d=1, V=V^{*}=1$ if $U=u_{0}$, and if $U=1, V=V^{*}=v_{0}$. Assume that the theorem holds for power products whose degree is less than $d$. By Lemma 3.3, two cases may be distinguished:

Case 1. $u_{n}$ is a factor of $U$,

Case 2. $u_{n}$ is not a factor of $U$.

In Case 1, consider $Q=P / u_{n}$ and $Q^{*}=P^{*} / u_{n}$. The degree of $Q$ and $Q^{*}$ is less than $d$ and the induction hypothesis may be applied to $Q$ and $Q^{*}$ yielding the conclusion that $V=V^{*}$. In Case 2, $v_{m}$ must be a factor of $V$ and $V^{*}$. The induction hypothesis may then be applied to $R=P / v_{m}$ and $R^{*}=P^{*} / v_{m}$, yielding the conclusion that $V / v_{m}=V^{*} / v_{m}$; and hence, $V$ and $V^{*}$ are the same.

THeOREM 3.5. If $A$ is a generalized $\alpha$-term of signature $(m, n)$, then

$$
A \equiv(-1)^{J} u_{0}^{m} v_{m}^{n},
$$

where $J$ is the u-weight of $A$.

Proof. The proof is by induction on $J$. If $J=0, A$ is an $\alpha$-term and the theorem is true. By the theorem of [3], assume that $A$ involves $u_{0}$ and let

$$
A=u_{0}^{a} v_{a}^{b} u_{b}^{c} \cdots
$$

By Lemma 1 of [3], with the roles of $u$ and $v$ interchanged, $i=a$, $j=b$, and $P=u_{0}^{a} v_{a}^{b-1}$,

$$
A \equiv-u_{0}^{a} v_{a}^{b-1} u_{b-1} v_{a-1} u_{b}^{c-1} \cdots .
$$

The term on the right is a generalized $\alpha$-term and has $u$-weight one less than the $u$-weight of $A$. Using the induction hypothesis, the proof may be completed. 
Since $u_{0}^{m} v_{m}^{n}$ is not in [uv] by [1], the following remark summarizes this section.

THEOREM 3.6. No generalized $\alpha$-term is in [uv].

4. A reduction theorem. In ([2], p. 429), D. G. Mead has several theorems for $\left[y^{2}\right]$, which, for practical purposes, reduce the degree of the power product under consideration. Similar results may be developed for [uv], with minor complications due to the asymmetrical definition of an $\alpha$-term. With the help of the symmetry theorems of [4], or [3], proper reduction theorems may be obtained.

For the rest of the paper, adopt the notation of [2]. Let $P$ be any power product of excess weight zero and $A$ the unique $\alpha$-term of the same weight and signature as $P$. The $M$-congruence $P \equiv c A$ is also written $m(P)=c$.

Lemma 4.1. Let $P=u_{i_{1}} \cdots u_{i_{m}} v_{j_{1}} \cdots v_{j_{n}}$. Then

$$
\begin{aligned}
& m(P)=m\left(u_{0} u_{i_{1}} \cdots u_{i_{m}} v_{j_{1}+1} v_{j_{2}+1} \cdots v_{j_{n}+1}\right) \\
& m(P)=(-1)^{m} m\left(v_{0} u_{i_{1}+1} u_{i_{2}+1} \cdots u_{i_{m}+1} v_{j_{1}} \cdots v_{j_{n}}\right) .
\end{aligned}
$$

Proof. The proof of part 1 is the same as that of Lemma 1, p. 429 , [2]. For part 2, the proof of part 1 with the $u_{i}$ and $v_{j}$ interchanged may be used, with the symmetry theorem of [4] or [3].

5. The main theorem. By Levi's theorem $\left(u_{i} v_{j}\right)^{t} \equiv 0$ for $t=$ $i+j+1$. For $i=0, u_{0}^{j} v_{j}^{j}$ is an $\alpha$-term and not congruent to zero, so that $1+j$ is the smallest power of $u_{0} v_{j}$ in $[u v]$. We conjecture that, in general, the smallest power of $u_{i} v_{j}$ in $[u v]$ is $i+j+1$. Theorem 5.1 implies that the conjecture is true for $i=1$.

THEOREM 5.1.

$$
\left(u_{1} v_{j}\right)^{1+j} \equiv(-1)^{1+j}(1+j) !\left(u_{0} v_{1+j}\right)^{1+j},
$$

for $j \geqq 0$.

Proof. Equivalently we may show that

$$
m\left(u_{1} v_{j}\right)^{1+j}=(-1)^{1+j}(1+j) !, \quad \text { for } j \geqq 0 .
$$

The proof is by induction on $j$; since $u_{1} v_{0} \equiv-u_{0} v_{1}, m\left(u_{1} v_{0}\right)=-1$ and the theorem is true for $j=0$. Assume that

$$
m\left(u_{1} v_{t}\right)^{1+t}=(-1)^{1+t}(1+t) ! \quad \text { for } t<j .
$$


By Levi's theorem, $Q=u_{0} u_{1}^{j} v_{j}^{j+1} \equiv 0$, and hence, $Q^{\prime} \equiv 0$. Thus

$$
\left(u_{1} v_{j}\right)^{1+j}+2 j\left(u_{0} u_{1}^{j-1} u_{2} v_{j}^{j+1}\right)+(j+1)^{2} u_{0} u_{1}^{j} v_{j}^{j} v_{j+1} \equiv 0 .
$$

Applying Lemma 4.1 results in the equation,

$$
m\left(u_{1} v_{j}\right)^{1+j}=-2 j m\left(u_{1}^{j-1} u_{2} v_{j-1}^{j+\mathrm{r}}\right)-(j+1)^{2} m\left(u_{1}^{j} v_{j-1}^{j} v_{j}\right) .
$$

Since $\left(u_{1}^{j} v_{j-1}^{j-1}\right)^{\prime} \equiv 0$, we also have

$$
m\left(u_{1}^{j-1} u_{2} v_{j-1}^{j-1}\right)=-\frac{j(j+1)}{2 j} m\left(u_{1}^{j} v_{j-1}^{j} v_{j}\right) .
$$

Substituting (2) into (1) yields,

$$
m\left(u_{1} v_{j}\right)^{1-j}=-(1+j) m\left(u_{1}^{j} v_{j-1}^{j} v_{j}\right) .
$$

Apply the induction hypothesis to the term on the right, to complete the proof.

6. More general results. Using the same methods, more general results may be obtained.

THEOREM 6.1. For $j \geqq 0, \quad k>j-1 \geqq 0, \quad l \geqq j-1 \geqq 0$

$$
m\left(v_{j} u_{1}^{k} v_{k}^{l} u_{l-1-j}\right)=(-1)^{k-l-j \div 1}\left\{j !\left(\begin{array}{c}
k \\
j
\end{array}\right)\left(\begin{array}{l}
l \\
j
\end{array}\right)+(j-1) !\left(\begin{array}{c}
k \\
j-1
\end{array}\right)\left(\begin{array}{c}
l \\
j-1
\end{array}\right)\right\} .
$$

Proof. The proof is by induction on the triple $(j, k, l)$, and a useful equation is derived first. Differentiate each of the following congruences, for $j \geqq 1, k \geqq j, l \geqq j-1$ :

$$
\begin{aligned}
& v_{j-1} u_{1}^{k} v_{k}^{l} u_{l+1-j} \equiv 0 \\
& u_{0} v_{j} u_{1}^{k} v_{k+1}^{l} u_{l+1-j} \equiv 0 .
\end{aligned}
$$

The resulting congruences yield, after applying Lemma 4.1, the equations:

$$
\begin{aligned}
m\left(v_{j-1} u_{1}^{k-1} u_{2} v_{k}^{l} u_{l+1-j}\right)= & -\frac{j}{2 k} m\left(v_{j} u_{1}^{k} v_{k}^{l} u_{+1-j}\right) \\
& -\frac{l(k+1)}{2 k} m\left(v_{j-1} u_{1}^{k} v_{k+1}^{l-1} u_{l+1-j}\right) \\
& -\frac{l+2-j}{2 k} m\left(v_{j-1} u_{1}^{k} v_{k}^{l} u_{l+2-j}\right) ;
\end{aligned}
$$

(4) $\quad m\left(v_{j-1} u_{1}^{k-1} u_{2} v_{k}^{l} u_{l+1-j}\right)=-\frac{1}{2 k} m\left(v_{j} u_{1}^{k+1} v_{k+1}^{l} u_{l+1-j}\right)$ 


$$
\begin{aligned}
& -\frac{(j+1)}{2 k} m\left(v_{j} u_{1}^{k} v_{k}^{l} u_{l+1-j}\right) \\
& -\frac{l(k+2)}{2 k} m\left(v_{j-1} u_{1}^{k} v_{k+1}^{l-1} u_{l+1-i j}\right) \\
& -\frac{(l+2-j)}{2 k} m\left(v_{j-1} u_{1}^{k} v_{k}^{l} u_{l+2-j}\right) .
\end{aligned}
$$

Combining (3) and (4), and representing $v_{j} u_{1}^{k} v_{k}^{l} u_{l+1-j}$ by $(j, k, l)$, the conclusion is:

$$
m(j, k+1, l)=-m(j, k, l)-l m(j-1, k, l-1) .
$$

If $m(p, q, r)=(-1)^{q+r-p+1}\left\{p !\left(\begin{array}{l}q \\ p\end{array}\right)\left(\begin{array}{l}r \\ p\end{array}\right)+(p-1) !\left(\begin{array}{c}q \\ p-1\end{array}\right)\left(\begin{array}{c}r \\ p-1\end{array}\right)\right\}$. then (5) implies

$$
\begin{gathered}
m(p, q+1, r)=(-1)^{(q+1)+r-p \div 1}\left\{p !\left(\begin{array}{c}
q+1 \\
p
\end{array}\right)\left(\begin{array}{c}
r \\
p
\end{array}\right)\right. \\
\left.+(p-1) !\left(\begin{array}{c}
q+1 \\
p-1
\end{array}\right)\left(\begin{array}{c}
r \\
p-1
\end{array}\right)\right\} .
\end{gathered}
$$

It remains to show that every triple $(j, k, l)$ with $k \geqq j-1$ can be constructed from triples where the formula is known to hold. Now first consider the cases $(\alpha) j=k$ and $(\beta) j=k+1$. By rearrangement of the power product $v_{j} u_{1}^{j-1} v_{j-1}^{l} u_{l+1-j}$,

$$
m(j-1, j-1, l-1)=m(j, j-1, l),
$$

so that if the formula is true for any case $(\alpha)$, then it is true for the next $(\beta)$ case, for all $l$.

Start with the case $(0,0, l), v_{0}^{l+1} u_{l+1}$. The formula is obviously true for this case (and indeed for $(0, k, l)$ ) with the convention that

$$
(j-1) !\left(\begin{array}{c}
k \\
j-1
\end{array}\right)\left(\begin{array}{l}
l \\
j-1
\end{array}\right)=0 \text {. }
$$

Then by (6) the formula must hold also for $(1,0, l)$. By (5)

$$
m(1,1, l)=-m(1,0, l)-\operatorname{lm}(0,0, l-1)
$$

and

$$
m(1,2, l)=-m(1,1, l)-l m(0,1, l-1) .
$$

In general, if the formula holds for $(j, j, l)$ (some particular $j$ and all $l)$, then it holds for $(j+1, j, l-1)$, all $l-1$. Using (5), the formula is then true for $(j+1, j+1, l)$. Therefore, the formula holds for all cases $\alpha$ and $\beta$; that is, if $k=j$ or $k=j-1$. 
Consider next the case $k=j+1$. For each $l$ by (5),

$$
m(1,3, l)=-m(1,2, l)-\operatorname{lm}(0,2, l-1) .
$$

The formula holds for $(0,2, l-1)$ since it holds for all $(0, k, l)$ and for $(1,2, l)$ as shown above; and, hence, the formula holds for $(1,3, l)$. In general, if the formula holds for $(j, j+p, l)$ and for $(j-1, j+p, l)$ for all $l$, then by (5)

$$
m(j, j \div p+1, l)=-m(j, j+p, l)-l m(j-1, j+p, l-1)
$$

and the formula holds for $k=j+p+1$. Thus the formula holds for all $k \geqq j$, and the proof of the theorem is complete.

\section{BIBLIOGRAPHY}

1. H. Levi, On the structure of differential polynomials and on their theory of ideals, Trans. Amer. Math. Soc. 51 (1942), 532-568.

2. D. G. Mead, Differential ideals, Proc. Amer. Math. Soc. 6 (1955), 420-432.

3. - A note on the ideal [uv], Proc. Amer. Math. Soc. 14 (1963), 607-608.

4. K. B. O'Keefe, A symmetry theorem for the differential ideal [uv], Proc. Amer. Math. Soc. 12 (1961), 654-657.

5. J. F. Ritt, Differential algebra, Amer. Math. Soc. Colloquium Publications, 33, (1950).

UNIVERSITY OF WASHINGTON 



\section{PACIFIC JOURNAL OF MATHEMATICS}

\section{EDITORS}

\section{H. SAMELSON}

Stanford University

Stanford, California

R. M. Blumenthal

University of Washington

Seattle, Washington 98105

\author{
*J. DugundJI \\ University of Southern California \\ Los Angeles, California 90007 \\ RICHARD ARENS \\ University of California \\ Los Angeles, California 90024
}

\section{ASSOCIATE EDITORS}
E. F. BECKENBACH
B. H. NeUManN
F. WolF
K. YosIDA

\section{SUPPORTING INSTITUTIONS}

UNIVERSITY OF BRITISH COLUMBIA
CALIFORNIA INSTITUTE OF TECHNOLOGY
UNIVERSITY OF CALIFORNIA
MONTANA STATE UNIVERSITY
UNIVERSITY OF NEVADA
NEW MEXICO STATE UNIVERSITY
OREGON STATE UNIVERSITY
UNIVERSITY OF OREGON
OSAKA UNIVERSITY
UNIVERSITY OF SOUTHERN CALIFORNIA

UNIVERSITY OF BRITISH COLUMBIA

UNIVERSITY OF CALIFORNIA

MONTANA STATE UNIVERSITY

NEW MEXICO STATE UNIVERSITY

OREGON STATE UNIVERSITY

OSAKA UNIVERSITY

UNIVERSITY OF SOUTHERN CALIFORNIA

\author{
STANFORD UNIVERSITY \\ UNIVERSITY OF TOKYO \\ UNIVERSITY OF UTAH \\ WASHINGTON STATE UNIVERSITY \\ UNIVERSITY OF WASHINGTON \\ AMERICAN MATHEMATICAL SOCIETY \\ CHEVRON RESEARCH CORPORATION \\ TRW SYSTEMS \\ NAVAL ORDNANCE TEST STATION
}




\section{Pacific Journal of Mathematics \\ Vol. 17, No. $1 \quad$ January, 1966}

Carlos Jorge Do Rego Borges, On stratifiable spaces ................ 1

Felix Earl Browder, Topological methods for non-linear elliptic equations of

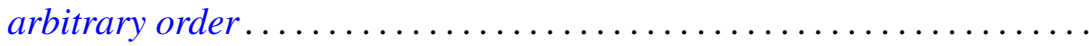

Gustave Choquet, Harry Corson and Victor Klee, Exposed points of convex

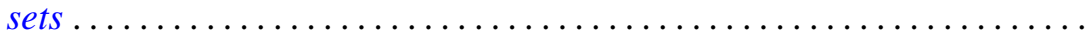

Phillip Emig, Remarks on the defect sum for a function meromorphic on an open Riemann surface ................................ 45

Ruth Goodman, A certain class of polynomials .................. 57

Sidney (Denny) L. Gulick, The bidual of a locally multiplicatively-convex

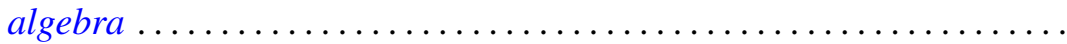

Eugene Carlyle Johnsen, Integral solutions to the incidence equation for finite projective plane cases of orders $n \equiv=2(\bmod 4) \ldots \ldots \ldots \ldots . .67$

Charles N. Kellogg, Centralizers and $H^{*}$-algebras .................. 121

Michael Lodato, On topologically induced generalized proximity relations. II .......................................... 131

P. H. Maserick, Half rings in linear spaces ..................... 137

Kathleen B O'Keefe, On a problem of J. F. Ritt .................... 149

Galen Lathrop Seever, Nonnegative projections on $C_{0}(X) \ldots \ldots \ldots \ldots$

Lawrence A. Shepp, Gaussian measures in function space ............ 167

Robert Charles Thompson, Classes of definite group matrices ........... 175 